\title{
Nebulate: Bridging Scale from Nanoparticles to Site
}

\author{
BEVERLY CHOE \\ Stanford University
}

Over the past 200 years, plastic has developed from a nascent wonder-material to a disposable and cheap resource. This installation is an investigation of the nature of plastic when our attitudes towards plastic have become so nebulous. Working with faculty in Architecture, Structural Engineering and the Material Sciences, students synthesized aspects of each discipline to produce Nebulate. They probed the physical and visual properties of sheet plastic to find new potentials for this mundane material.

The installation's structure was comprised of plastic bubbles: $1 / 8$ " thick PETG sheets formed and assembled into a translucent, cloud-like mass. Students developed overlapping clover-shaped panels to form balls of various sizes and forms. They heated and formed the sheets on semispherical molds, and then finished shaping the balls manually with heat guns. Through this process, each ball acquired a unique surface of depressions and undulations. Using plastic's malleability to structural advantage, students explored how surface deformation, through dimpling and curvature, increased the strength of the panels. The aggregated balls were evaluated with axial force diagrams and buckling strength analyses, determining rules for optimal relationships between the balls. Assembled together, the nebulous forms produced a lenslike space which both framed and distorted the surrounding site: the result of intertwined architectural, structural and material inquiries.

Students collaborated with a lab in the Material Sciences and Engineering Department, developing nanoparticle-based structural color for the panels, adding another layer of visual complexity to the piece while expanding the typical scale at which one designs. Structural color relies on the interaction of light with the nanostructure of a material rather than any chemical dye or pigment to give color. The size of these structures is typically smaller than the wavelength of visible light ( $400-800$ nanometers). At this micron scale, one can design structures with different shapes, sizes, and materials that absorb and scatter specific frequencies (colors) of light. Students used colloidal chemistry to synthesize gold nanoparticles in water. By modifying the ratio of the starting chemicals, students altered the diameter of the gold spheres from $\sim 10 \mathrm{~nm}$ to $\sim 20 \mathrm{~nm}$, which shifted the overall color from red to purple. The formation of the nanoparticle mixture at the microscale mirrored the deliberate spatial and structural formation of the larger piece.

\author{
JUN SATO \\ Tokyo University
}

Students then added polyvinyl alcohol, a polymer found in glues, to thicken the nanoparticle paint and facilitate application on the structure's plastic surface. Students applied the pinkish nanoparticle mixture in the structural dimples, producing an interplay between the material, spatial and structural dimensions of the piece. In aggregate, the largescale pavilion form embodied ideas of plasticity through its ebbs, flows and mutations, but also between the multiple disciplines at play. When the exhibition period ended, students reconfigured the modules into furniture elements which were dispersed to dorms across campus. Through these reciprocal processes, Nebulate was able to operate from the micron scale to the human scale, and from the scale of site to beyond. 

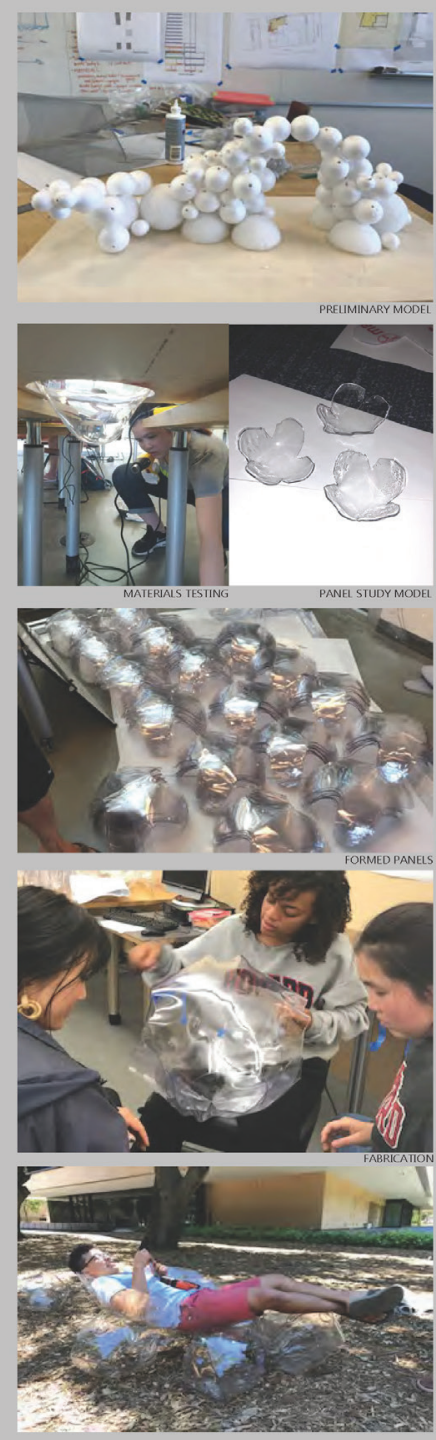

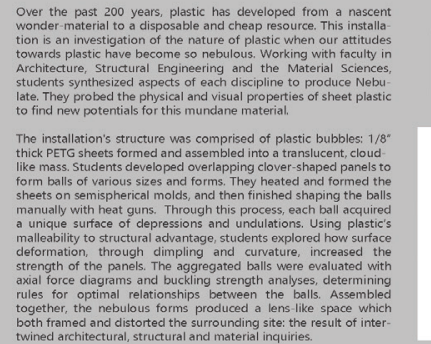

DEBULITE
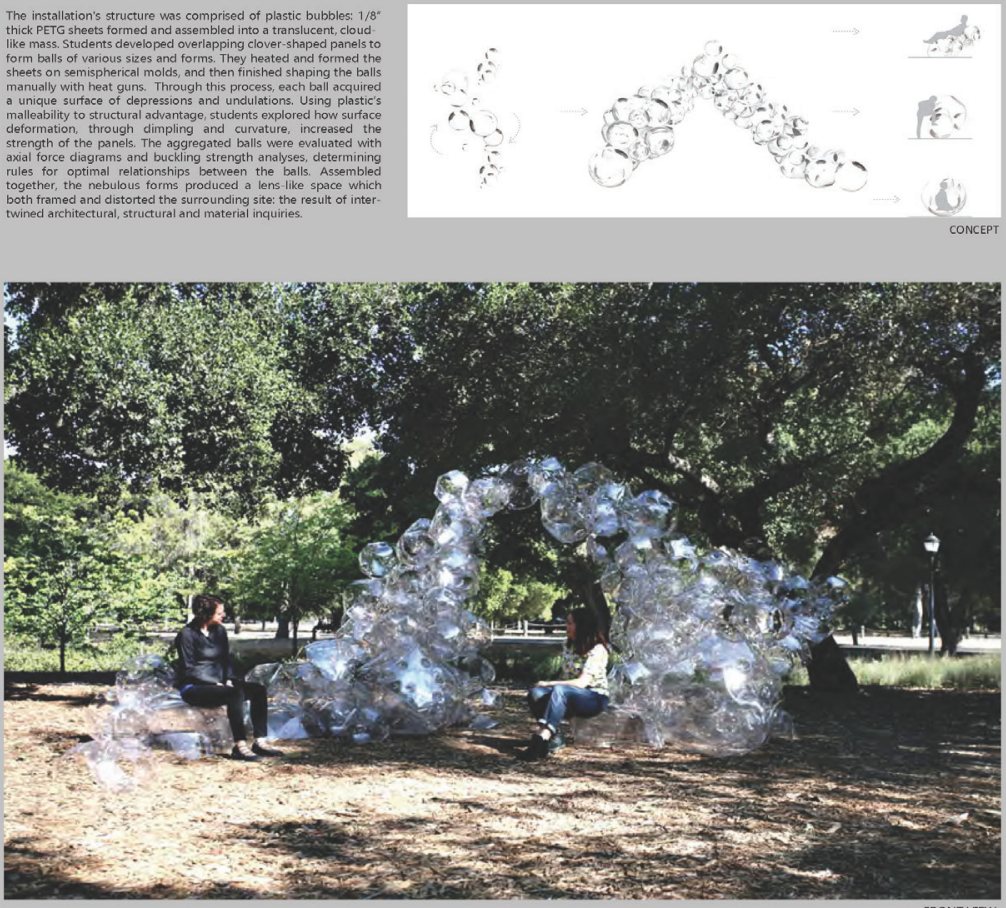

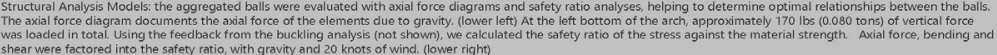
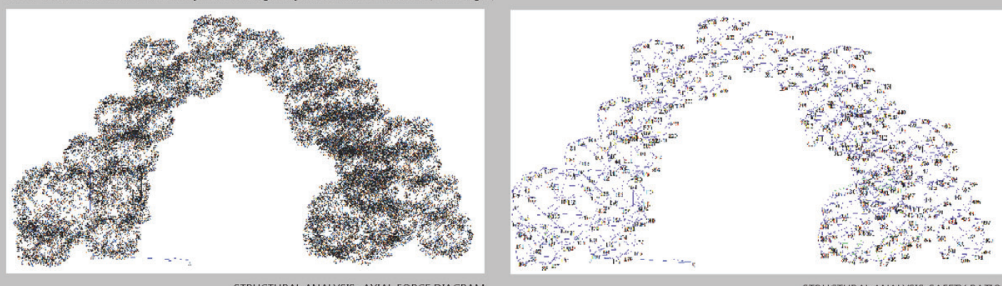
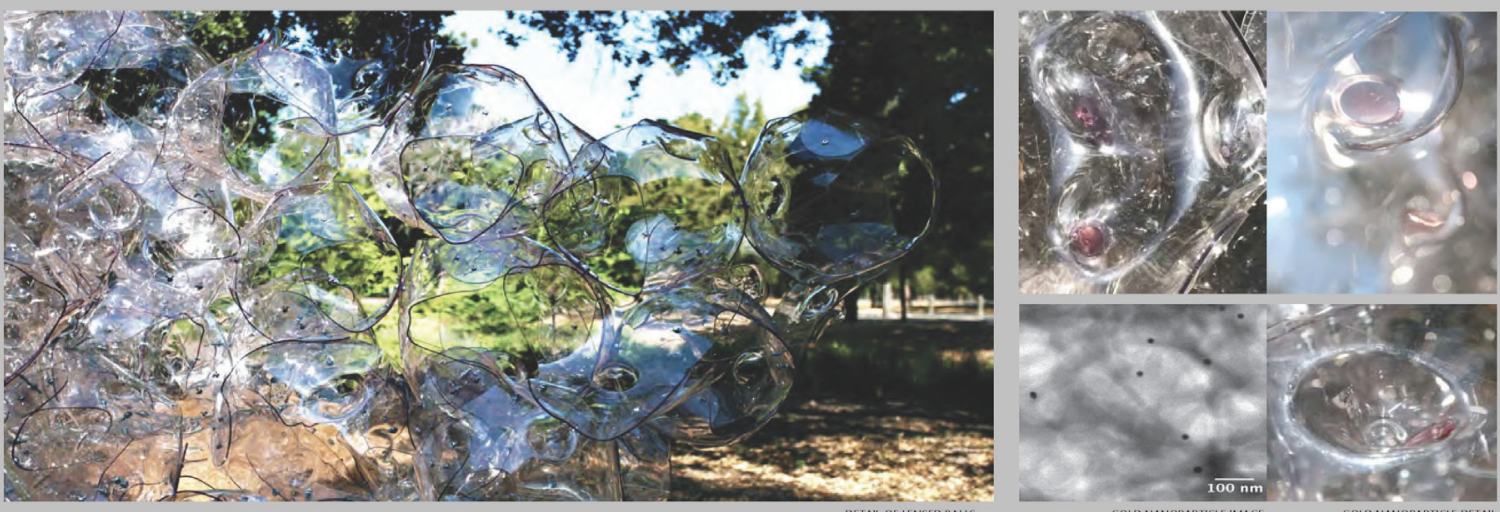

GOID NANOPARTICIE IMAGE,
RECORDED WIIH HECIRON MLROSCOPE

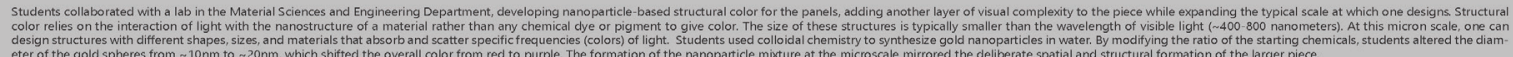

